\title{
Near infrared LED: an emerging technology on the treatment of stroke
}

\begin{abstract}
New research in the area of near infrared light has created interesting avenues of coadjuvant therapy to help with decreasing the effects of cerebrovascular accidents and traumatic brain injuries. With increasing knowledge of near infrared Light Emitting Diodes there has been an increase in available and cost effective ways the technology can be used. Near infrared technology can be used to create an oxygen molecular analysis, neuroimaging, and intervention therapy to help with diagnosis and treatment of disease. The use of near infrared light has shown accelerated tissue repair after an injury. Even thought there have been advances with the use of near infrared light technologies, more studies should be done on the most appropriate wavelength. This opinion cites literature in animal and human studies that shows the benefits of Light Emitting Diode therapy.
\end{abstract}

Cerebrovascular disease is the third leading cause of death in the western hemisphere. The improvement of pharmaceutical interventions has aided in the decline of deaths but still more research is needed. Near infrared is an emerging technology available, obtained through Light Emitting Diode or laser, for the therapy of cerebrovascular disease and traumatic brain injuries. Near infrared is a therapy that could be used adjunct to other therapies, with an interesting outcome.
Volume I Issue 6 - 2014

\author{
Manuel Dujovny,' Onyekachi Ibe,' Pablo \\ Sosa, ${ }^{2}$ Erin Morency ${ }^{3}$ \\ 'Department of Neurosurgery, Department of Electrical \\ Engineering, Wayne State University, USA \\ ${ }^{2}$ Department of Neurosurgery, University of Cuyo, Argentina \\ ${ }^{3}$ Department of Nursing, Oakland University, USA
}

\begin{abstract}
Correspondence: Manuel Dujovny, Department of
Neurosurgery, Wayne State University, 196 Long Lake Shore Dive, Bloomfield Hills, MI 4832, USA, Tel 248-758-9662, Fax 248758-9667,Email manueldujovny@hotmail.com
\end{abstract}

Received: September 12, 2014 | Published: November 01, 2014

\section{Opinion}

Near infrared is a subdivision in the infrared spectrum between the range of 800 nanometers and 2,500 nanometers wavelength. There are no exact numbers on these wavelengths. Near Infrared is found on the right outermost of the visible light band of the electromagnetic spectrum. Visible Light near infrared emits irradiation in the spectrum of the human eye and its electromagnetic spectrum is in the range of 380 nanometers- 760 nanometers, which can be detected by the human eye. Infrared light is an electromagnetic radiation that has a wavelength longer than a visible light. The technique to obtain near infrared using Light Emitting Diodes has made the equipment easier to manipulate, more accessible, and easier to operate. Electromagnetic waves are waves of energy having a frequency within the electromagnetic spectrum and propagated as a periodic disturbance of the electromagnetic field. When an electric charge oscillates or accelerates not all photons are visible to the human eye. What we see as light is only a minute range of the spectrum of electromagnetic waves associated with photons. The entire spectrum includes radio waves, infrared radiation, visible light, ultraviolet rays, $\mathrm{x}$-rays, gamma rays, and cosmic radiation. The photons of different regions of the electromagnetic spectrum vibrate differently and have different amounts of energy. Examples of near infrared devices are night vision goggles, digital cameras, remote controls, etc. ${ }^{1}$ Near infrared discovery is credited to Herschel during the 1800 s, but the industrial use of near infrared was not developed until the 1950s when the near infrared was used as an add-on unit to other optical devices with a different wavelength, such as the ultraviolet spectrometers. Today near infrared is used in a variety of clinical and medical applications such as molecular spectroscopy, photodynamic therapy, optic thermography, photobiomodulation, remote monitoring, thermal radiation (heat) and other optical devices.

Light Emitting Diodes were first developed in 1927 by Oleg Losev, a Russian scientist, who distributed his research in several scientific journals, but there was no use for it for several decades until 1955 when Rubin Braunstein found near infrared emission generated by the diode structures using gallium arsenide. In 1961, Robert Biard and Gary Pittman, from Texas Instrument, discovered that gallium arsenide could emit infrared radiation when an electrical current is applied to the diode. In 1962, Nick Holonyak, seen as the father of Light Emitting Diodes, developed the first visible spectrum (RED) Light Emitting Diodes while working with General Electric Company. George Crawford, a former student of Holonyak's, developed a (YELLOW) Light Emitting Diode and also increased the brightness of the red and orange Light Emitting Diodes to a factor of ten in $1972 .^{2}$ The remarkable scientific discovery of the Light Emitting Diode started out as an attempt to develop light that would allow for the growth of plants in space, which was proposed by Ronald W. Ignatius, the founder and chairman of the board at Quantum Devices Inc. in Barneveld, Wisconsin. The company's proposition was made at a meeting held by the Wisconsin Center for Space Automation and Robotics, a NASA-sponsored research center that was facilitating the commercialization of robotics, automation, and other advanced technologies. The proposal proved successful which led to the production of Astroculture3, a plant growth chamber that successfully incorporated this Light Emitting Diode light source, which has now flown on several space shuttle missions. According to Whelan et al. ${ }^{3}$ Light Emitting Diodes use longer wavelengths than the laser, and while the laser can pinpoint one location in the body the Light Emitting Diode can treat the entire body, which makes the light emitting diode useful in treating serious burns, crush injuries, and complications of cancer. Light Emitting Diode therapy equipment is versatile, portable, cost effective, easy to use and can be used in hospitals, medical offices, sport fields, or homes with minimal training required. ${ }^{4}$

\section{Cerebrovascular disease and stroke}

Reducing the oxygen concentration in the brain is one of the determinants in the pathophysiology of stroke. From this we can deduce that a monitoring of the concentration of this gas could help detect variations in real time. ${ }^{5}$ Dujovny et al. ${ }^{6}$ showed the value of oxygen concentration monitoring during carotid cavernous sinus 
fistula embolization and after the performance of cranioplasty defect. They also demonstrated the changes from prone to supine position. ${ }^{7}$ He also suggested the use of oxygen near infrared spectroscopy in any case of endovascular cerebral angiography. ${ }^{8}$ The use of the transcranial cerebral oximetry monitoring during heart surgery showed the significant cognitive improvement on the patient being monitored. ${ }^{9}$ One of the determining factors in the genesis of stroke is related to inflammatory processes in the arterial territory of the vessels that irrigate the brain. Jaffer et al. ${ }^{10}$ study in 2011, on a new 2D intravascular near infrared fluorescence imaging, shows high-resolution in vivo spatial mapping of arterial inflammation in coronary-sized arteries and also increase on inflammation-regulated cysteine protease activity in atheromata and stent-induced arterial injury. Near infrared has been effective in the detection of inflammation of the carotid plaque through photoluminescence, caused by indocyanine green, and endoscope. ${ }^{11}$ Near infrared Light Emitting Diodes produce cellular protection against hypoxia and reoxygenated-induced cardiomyocyte injury. ${ }^{12}$ During neurosurgical procedures, especially vascular procedures, near infrared light, associated with indocyanine green and the microscope integrated near infrared video angiography, shows the vascular anatomic structure for the aneurysm clipping to reduce the incidence of post operative ischemic complications. Luar et al showed the variation of oxygen concentration vasospasm after cerebral hemorrhage and in 1994 an alternative technique for the transcrainal oximetry in acute subarachnoid hemorrhage was also demonstrated. ${ }^{13}$ Intravascular near infrared fluorescence catheters may offer a new in vivo method to detect high-risk coronary plaques and to assess novel atherosclerosis biologics. ${ }^{14}$ Indocyanine green videographer can be used with patients undergoing carotid endarterectomy to provide instantaneous information on the carotid plaque and the patency of the arterial vessel. ${ }^{15}$

It is known that when a stroke occurs many changes at the cellular level are generated. ${ }^{16}$ Some of these changes are decreased adenosine trisphosphate, nitric oxide, and the activity of the cytochrome c oxidase. The cytochrome c oxidase activity, adenosine trisphosphate, nitric oxide, neuron, and neuroglial cell growth can be stimulated by near infrared light. ${ }^{17}$ In 2012 , Choi et al. ${ }^{18}$ showed the effects of $710 \mathrm{~nm}$ visible light irradiation on neuronal protection and neuronal outgrowth in an in vitro stroke model in rat. The cytochrome c oxidase activity in cultured neuronal cells improved the ischemic tissue healing also the near infrared accelerates the arrival of the reactive astrocytes, microglia, macrophage, nitric oxide, and increased growth factors and neurotransmitters. ${ }^{19}$ Wong-Riley et al..$^{20}$ increased cytochrome c oxidase activity by stimulation with Light Emitting Diodes in primary neuronal cultures and determined that the photobiomodulation increases the activity of cytochrome c oxidase. According to Lapchak and De Taboada ${ }^{21}$ transcrainal near infrared laser therapy improves behavioral outcome following embolic stroke. It can also increase the cortical adenosine-5-triphosphate in embolized rabbit and acute ischemic stroke using $808 \mathrm{~nm}$ continuous wave and pulsed wave. There has also been observed a decrease in brain edema confirmed by magnetic resonance imaging with clinical improvements on experimental animals. ${ }^{22}$ Sharma et al. ${ }^{23}$ demonstrated that stimulation of neurons with near infrared light modulated concentrations of nitric oxide and reactive oxygen species in cultured neurons. Near infrared of $808 \mathrm{~nm}$ irradiation can help improve residual cerebral blood flow and reduce the amount of apoptotic cells in the hippocampus and also increase the cerebral nitric oxide concentration in mice. ${ }^{24}$ Near infrared Fluorescence diagnostic imaging can be used for the detection of fibrin depositions in an ischemic mouse brain. ${ }^{25}$ As seen, numerous studies in animals and cell cultures demonstrate the positive effects of photobiomodulation with Light Emitting Diode near infrared light.
Some studies have been conducted in humans, who have shown the potential therapeutic effects of stimulation with near-infrared light, but more studies are still needed. A New Treatment Strategy of NeuroThera Effectiveness and Safety Trial, using the infrared laser therapy, showed initial safety and effectiveness for the treatment of ischemic stroke in humans when initiated within 24 hours of stroke onset. ${ }^{26}$ A randomized double-blind study by Zivin et al. ${ }^{27}$ in 2009 concluded in NEST-2, of 660 patients with acute ischemic stroke, $36.3 \%$ of patients treated with transcranial near infrared laser technology within 24 hours had a favorable outcome as compared to $30.9 \%$ demonstrated to be safe, but the data did not meet the statistical significance for efficacy, further trials are needed. Transcranial near infrared therapy has been used for neuroprotective treatment of acute ischemic stroke patients. ${ }^{28}$ The neuro imaging used to map brain molecular imaging events after cerebrovascular accidents, or traumatic brain injury, has been demonstrated on the evaluation of a stroke patient after recovery and rehabilitation. ${ }^{29,30}$ traumatic brain injury, resulting in impaired cognitive function, treated with near infrared Light Emitting Diodes of $633 \mathrm{~nm}$ and $870 \mathrm{~nm}$ saw cognitive function improvement after the near infrared Light Emitting Diode radiation..$^{31}$ Increases in cerebral blood flow have been observed after near infrared Light Emitting Diode radiation in a patient in a persistent vegetative state. ${ }^{32}$

Early utilization of dimethyl silfoxide has shown the decrease of cerebral infarction or decrease of the free radical and the increase of Adenosine Triphosphate..$^{33}$ The mitochondria generate the Adenosine Triphosphate. The mitochondria will improve the electrical channel for Adenosine Triphosphate and Adenosine Diphosphate and these can induce stimulation on other neurotransmitters and growth factors. It is known that the near infrared induces the stimulation of the cell mitochondria and stimulates the Adenosine Triphosphate and Adenosine Diphosphate energy channel and other processes. Original research on near infrared effects on infarctions shows the improvement of Adenosine Triphosphate (energy channel). ${ }^{34}$

The Light Emitting Diode is a solid semiconductor light source that emits infrared and visible light when charged with an electric current and is highly resistant to shock. The new technology of the Light Emitting Diode phototherapy devices are economically accessible, no training is needed, easy to use, activated by electrical outlet or battery, portable, and can be applied several times throughout the day anywhere, including the patient's home. If used appropriately the Light Emitting Diode phototherapy devices are safe, but it should not be aimed into the eye, used during early pregnancy, or used with known cancer related pain. Light Emitting Diode technology phototherapy devices needs to be considered in the future for new research on cerebrovascular disease.

\section{Acknowledgments}

None.

\section{Conflicts of interest}

The Author declares that there is no conflict of interest regarding the publication of this paper, nor received any financial compensation or other consideration in connection with the preparation and/or publication of the this manuscript.

\section{References}

1. Seling Duan. Light emitting diodes analysis on construction, material, uses and socioeconomic impact. San Jose State University. 2002. 
2. Held G. Introduction to light emitting diode technology and applications. CRC Press Science. 2008;1-192.

3. Whelan HT, Smits RL, Buchman EV, et al. Effect of NASA lightemitting diode irradiation on wound healing. J Clin Laser Med Surg. 2001;19(6):305-314.

4. Enwemeka CS, Parker JC, Dowdy DS, et al. The efficacy of low-power laser in tissue repair and pain control: a meta-analysis study. Photomed Laser Surg. 2004;22(4):323-329.

5. Arnulphi MC, Alaraj A, Slavin KV. Near infrared technology in neuroscience; past, present, and future. Neurol Res. 2009;31(6):605614.

6. Dujovny M, Fernandez P, Alperin N, et al. Post-cranioplasty cerebral spinal fluid hydrodynamic changes: Magnetic resonance imaging quantitative analysis. Neurol Res. 1997;19(3):311-316.

7. Misra M, Alp MS, Dujovny M, et al. Near-infrared spectroscopy. $J$ Neurosurg. 1996;85(2):363-364.

8. Dujovny M, Misra M, Alp MS, et al. Carotid-cavernous fistula and jugular venous oxygen saturation. $J$ Neurosurg. 1996;84(4):713.

9. Slater JP, Guarino T, Stack J, et al. Cerebral oxygen desaturation predicts cognitive decline and longer hospital stay after cardiac surgery. Ann Thorac Surg. 2009;87(1):36-44.

10. Jaffer FA, Calfon MA, Rosenthal A, et al. Two-dimensional intravascular near-infrared fluorescence molecular imaging of inflammation in atherosclerosis and stent-induced vascular injury. J Am Coll Cardiol. 2011;57(25):2516-2526.

11. Calfon MA, Rosenthal A, Mallas G, et al. In vivo near infrared fluorescence (NIRF) intravascular molecular imaging of inflammatory plaque, a multimodal approach to imaging of atherosclerosis. J Vis Exp. $2011 ;(54): 2257$

12. Zhang R, Mio Y, Pratt PF, et al. Near infrared light protects cardiomyocytes from hypoxia and reoxygenation injury by a nitric oxide dependent mechanism. J Mol Cell Cardiol. 2009;46(1):4-14.

13. Luer MS, Dujovny M, Slavin KV, et al. Regional cerebral oxygen saturation during intra-arterial papaverine therapy for vasospasm: Case report. Neurosurgery. 1995;36(5):1033-1036.

14. Calfon MA, Vinegoni C, Ntziachristos V, et al. Intravascular nearinfrared fluorescence molecular imaging of atherosclerosis: toward coronary arterial visualization of biologically high-risk plaques. $J$ Biomed Opt. 2010;15(1):011107.

15. Pennekamp CW, Bots ML, Kappelle LJ, et al. The value of nearinfrared spectroscopy measured cerebral oximetry during carotid endarterectomy in perioperative stroke prevention. Eur J Vasc Endovasc Surg. 2009;38(5):539-545.

16. Caplan JM, Sankey E, Yang W, et al. Impact of Indocyanine Green Videoangiography on Rate of Clip Adjustments Following Intraoperative Angiography. Neurosurgery. 2013;75(4):437-444.

17. Li JY, Ueda H, Seiyama A, et al. A near-infrared spectroscopic study of cerebral ischemia and ischemic tolerance in gerbils. Stroke. 1997;28(7):1451-1456.

18. Choi DH, Lee KH, Kim JH, et al. Effect of $710 \mathrm{~nm}$ visible light irradiation on neurite outgrowth in primary rat cortical neurons following ischemic insult. Biochem Biophys Res Commun. 2012;422(2):274-279.
19. Ball KA, Nelson AW, Foster DG, et al. Nitric oxide produced by cytochrome c oxidase helps stabilize HIF-1 $\alpha$ in hypoxic mammalian cells. Biochem Biophys Res Commun. 2012;420(4):727-732.

20. Riley MTW, Liang HL, Eells JT, et al. Photobiomodulation directly benefits primary neurons functionally inactivated by toxins: role of cytochrome c oxidase. J Biol Chem. 2005;280(6):4761-4771.

21. Lapchak PA, De Taboada L. Transcranial near infrared laser treatment (NILT) increases cortical adenosine-5'-triphosphate (ATP) content following embolic strokes in rabbits. Brain Res. 2010;1306:100-105.

22. Oron A, Oron U, Streeter J, et al. Near infrared transcranial laser therapy applied at various modes to mice following traumatic brain injury significantly reduces long-term neurological deficits. J Neurotrauma. 2012;29(2):401-407.

23. Sharma SK, Kharkwal GB, Sajo M, et al. Dose response effects of 810 $\mathrm{nm}$ laser light on mouse primary cortical neurons. Lasers Surg Med. 2011;43(8):851-859.

24. Uozumi Y, Nawashiro H, Sato S, et al. Targeted increase in cerebral blood flow by transcranial near-infrared laser irradiation. Lasers Sura Med. 2010;42(6):566-576

25. Zhang Y, Fan S, Yao Y, et al. In vivo near-infrared imaging of fibrin deposition in thromboembolic stroke in mice. PLoS One. 2012;7(1):e30262.

26. Lampl Y, Zivin JA, Fisher M, et al. Infrared laser therapy for ischemic stroke: a new treatment strategy. Stroke. 2007;38(6):1843-1849.

27. Zivin JA, Albers GW, Bornstein N, et al. Effectiveness and safety of transcranial laser therapy for acute ischemic stroke. Stroke. 2009;40(4):1359-1364.

28. Schellinger PD, Kohrmann M. Near-infrared laser treatment of acute stroke: from bench to bedside. Nervenarzt. 2012;83(8):966-974.

29. Mihara M, Yagura H, Hatakenaka M, et al. Clinical application of functional near-infrared spectroscopy in rehabilitation medicine. Brain Nerve. 201062(2):125-132.

30. Lapchak PA. Taking a light approach to treating acute ischemic stroke patients: transcranial near-infrared laser therapy translational science. Ann Med. 2010;42(8):576-586.

31. Naeser MA, Saltmarche A, Krengel MH, et al. Improved cognitive function after transcranial, light-emitting diode treatments in chronic, traumatic brain injury: two case reports. Photomed Laser Surg. 2011;29(5):351-358.

32. Nawashiro H, Wada K, Nakai K, et al. Focal increase in cerebral blood flow after treatment with near-infrared light to the forehead in a patient in a persistent vegetative state. Photomed Laser Surg. 2012;30(4):231233

33. Chao JC, Medeiros DM, Davidson J, et al. Low levels of ATP Synthase and cytochrome $\mathrm{c}$ oxidase subunit peptide from hearts of copperdeficient rats is not altered by the administration of dimethyl sulfoxide. J Nutr. 1994;124(6):789-803.

34. Li X, Hou J, Wu B, et al. Effects of platelet-rich plasma and cell coculture on angiogenesis in human dental pulp stem cells and endothelial progenitor cells. J Endod. 2014;pii:S0099-2399(14)00658-X. 